\title{
Amelanotic acral melanoma misdiagnosed as verruca plantaris*
}

\author{
Wei Deng ${ }^{1}$, Ruixing Yu ${ }^{1}$, Yong Cui ${ }^{1}$, Zhancai Zheng ${ }^{1}$ \\ DOI: http:/ / dx.doi.org/10.1590/abd1806-4841.20197568
}

\begin{abstract}
We report a case of amelanotic acral melanoma in a 42-year-old Chinese woman. Ten months previously the patient found a $2-\mathrm{cm}$ asymmetric erythematous macular plaque on her left sole. The lesion was diagnosed as verruca plantaris by every physician the patient consulted. One month ago, an enlarged lymph node was detected in the left groin, which biopsy reported as metastatic melanoma. Dermoscopy suggested verruca plantaris, and positron emission tomography (PET) revealed increased glucose metabolism in the macular plaque. Finally, biopsy of the plaque revealed amelanotic melanoma. Misdiagnosis and diagnostic delay are usually associated with poorer patient outcomes. Awareness of atypical presentations of acral melanoma is thus important for decreasing misdiagnosis rates and improving patient outcomes.
\end{abstract}

Keywords: Lymphatic metastasis; Melanoma, amelanotic; Warts

\section{INTRODUCTION}

Amelanotic melanoma (AM) is a degenerative development of melanoma, composed of cells derived from melanocytes but not forming melanin granules. It accounts for $2 \%-20 \%$ of melanoma lesions. ${ }^{1}$ Early lesions are not typical, usually asymmetric pink nodules with uniform color, unclear boundaries, and often slight pigmentation on the edge. In later stages they can infiltrate and grow as red plaques, granulomatous nodules, or ulcers. ${ }^{2}$ Clinical and pathological diagnosis of AM is difficult, given its different clinical presentations and the fact that it may mimic non-melanocytic lesions. Plantar melanoma exhibits a higher misdiagnosis rate relative to other anatomical sites. ${ }^{3}$ Misdiagnosis and delay in diagnosis are statistically associated with poorer patient outcomes. Awareness of atypical presentations of acral melanoma is thus important for decreasing misdiagnosis rates and improving patient outcomes.

\section{CASE REPORT}

A 42-year-old Chinese female, otherwise healthy, presented with a 10-month history of an asymmetric $2-3$ cm erythematous macular papule on her left sole that had increased in size with accompanying minor tenderness (Figure 1). In the previous 6 months the lesion had been diagnosed as verruca plantaris by 5 dermatologists, all of whom had recommended liquid nitrogen cryotherapy, which the patient had not done. She had used frequent corn plasters and 2 months previously had pared down the thick cuticles on the lesion's surface. One month previously, an enlarged lymph node was detected in her left groin which biopsy in the local hospital report- ed as metastatic melanoma (Figure 2). She came to our outpatient department and underwent dermoscopic examination of the lesion, which suggested verruca plantaris. For further verification, a positron emission tomography(PET) scan was performed, showing elevated glucose metabolism in the left groin and in the macular papule (Figure 1). One week previously she was admitted for further treatment. Physical examination revealed a 2 to $3 \mathrm{~cm}$ pink macular papule on the pressure points of the left sole, surrounded by a firm, horny ring. Irregular pigmented spots measuring approximately $0.5 \mathrm{~cm}$ were visible on the edge of the ring, and the top of the nodule had been pared down, showing small black dots representing dilated capillary loops (Figure 1). Full metastatic workup was performed, and PET scan was positive for left inguinal lymph node involvement and negative for metastases to other lymph nodes and organs. She was otherwise fit and well, had no significant medical history, and was not on any regular medications. An excisional biopsy showed an AM with Breslow thickness of $2 \mathrm{~mm}$, dermal mitotic rate $>1 / \mathrm{mm}^{2}$, Clark level papillary dermis, and presence of brisk tumor-infiltrating lymphocytes. No microsatellitosis, regression, angiolymphatic invasion, or neurotropism were detected (Figure 2). On immunohistochemical analysis, S100, HMB45, and Melan-A were positive and Ki67 was 40\% positive (Figure 3). Cytogenetic examination was negative for gene BARF V600E mutation. The case was diagnosed as metastatic amelanotic acral melanoma clinical stage III and pathologic stage IIIC (T2aN1bM0). A re-excision with a $2 \mathrm{~cm}$ margin showed no residual tumor, and complete lymph node dissection was performed.

\footnotetext{
Received 21 August 2017.

Accepted 21 February 2018.

* Work performed at the China-Japan Friendship Hospital, Beijing, China.

Financial support: None.

Conflict of interest: None.

1 Department of Dermatology and Venereology, China-Japan Friendship Hospital, Beijing, China.
}

MAILING AdDRESS:

Wei Deng

E-mail: dengwei1991730@163.com

C2019 by Anais Brasileiros de Dermatologia 


\section{DISCUSSION}

We present a case of stage IIIC metastatic AM that posed a diagnostic challenge because of its clinical presentation. The patient had been in her local hospital several times, and the lesion had been diagnosed as verruca plantaris every time. Until the enlarged lymph node biopsy showed metastatic melanoma, the attending physicians were still in doubt whether the nodule was the primary lesion, due to its verruca plantaris appearance and the fact that der-
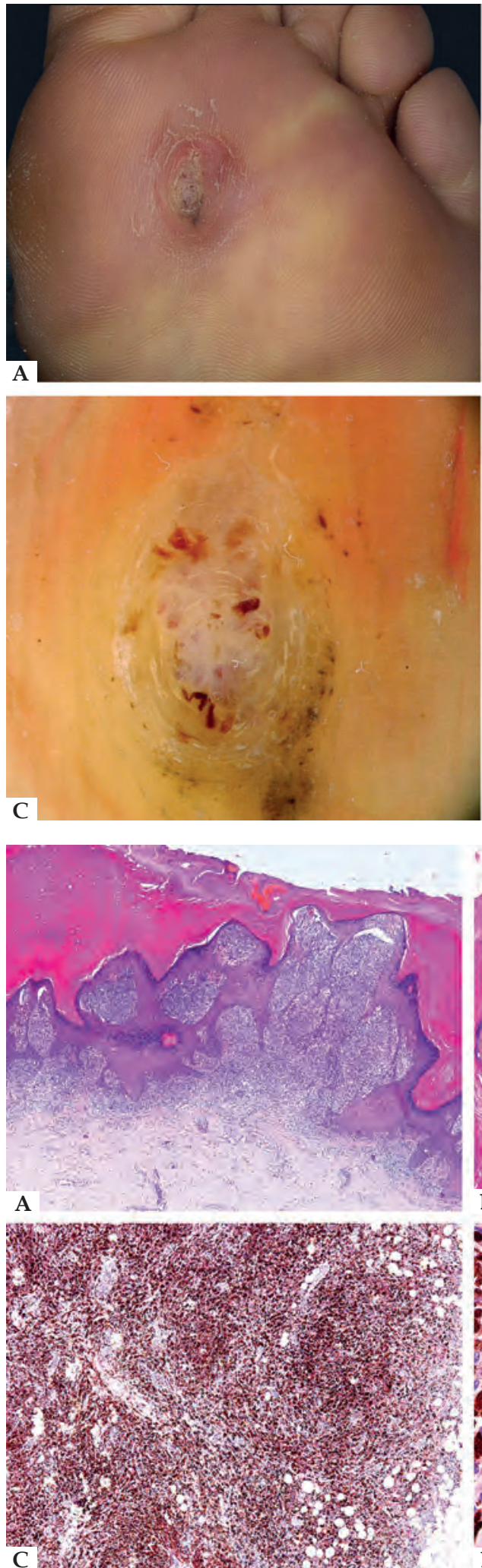
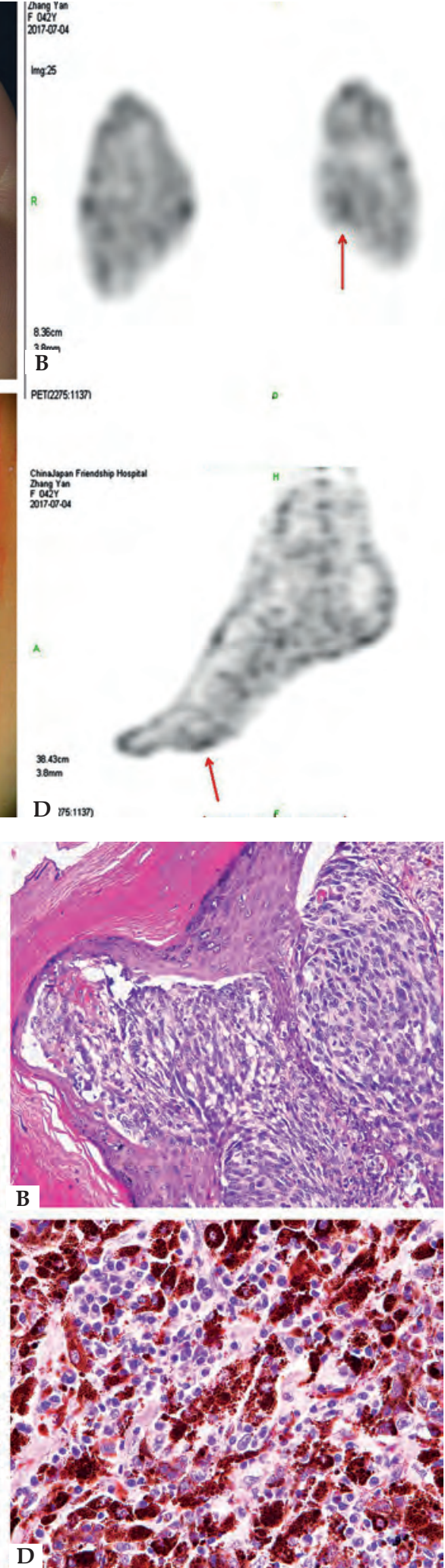

Figure 1: A: Lesion on the sole; C: Linear bleeding seen on dermoscopy, with large areas of unstructured space; $\mathbf{B}$ and D: PET scan showed elevated glucose metabolism in the macular papule on the left sole

Figure 2: A and B: Histopathology: macular papule showed tumor cells arranged as nests in dermo-epidermal junction and dermis, and no obvious melanin was observed; $C$ and $D$ : histopathology: left inguinal lymph nodes revealed dense infiltration of tumor cells with rich melanin granules in cytoplasm (hematoxylin \& eosin, A, x40; B, x200; C, x40; D, x200) 

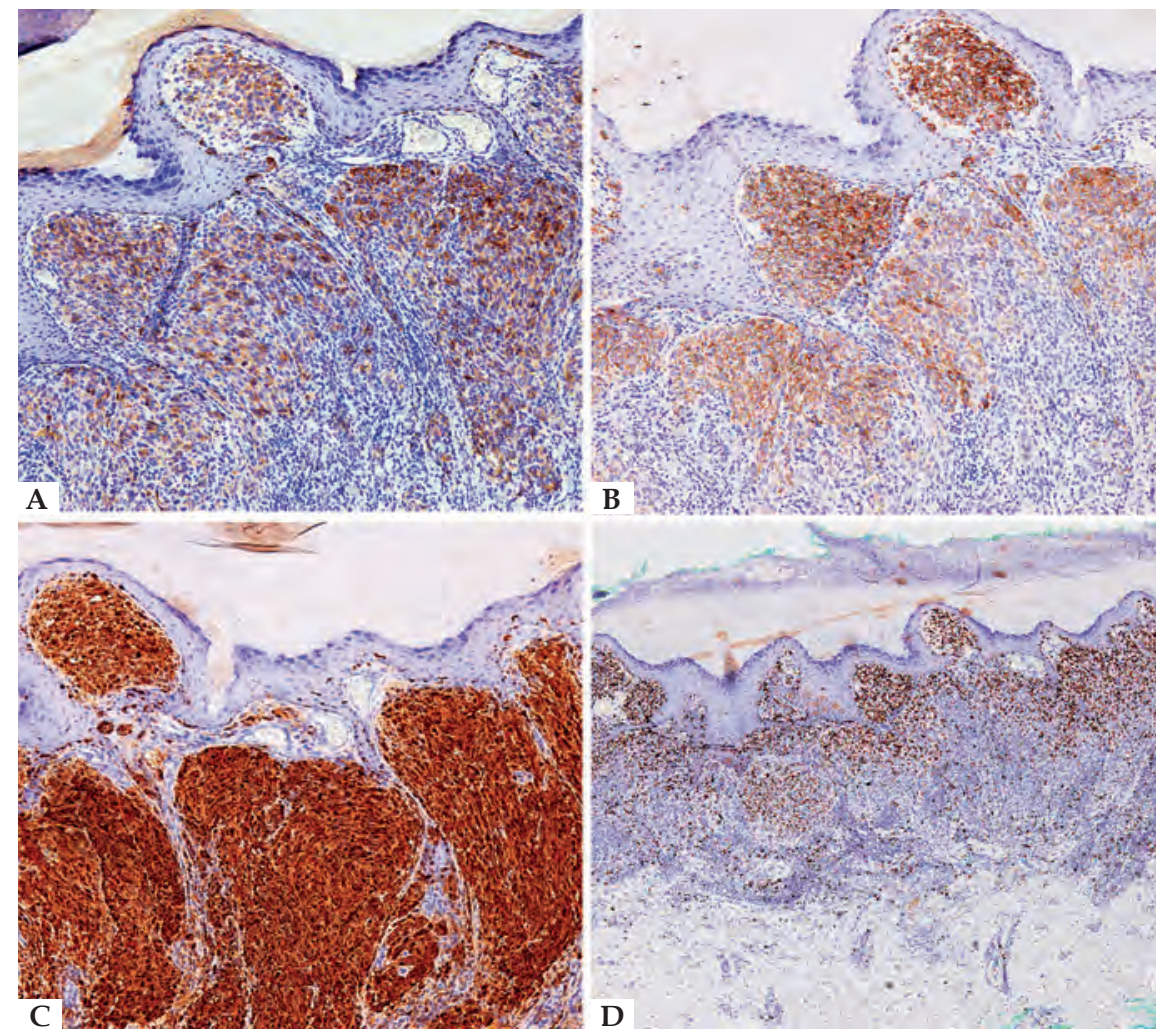

Figure 3: A - Tumor cells positive for Melan-A; B - Tumor cells positive for HMB-45; C - Tumor cells positive for S-100; D - Tumor cells $40 \%$ positive for Ki67; (A, Melan-A x100; B, HMB-45 x100, ; C, S-100 x100 ; D, Ki67 x40) moscopy of the lesion also suggested verruca plantaris. Biopsy of the nodule finally solved the riddle, showing that it was a malignant melanoma. Misdiagnosis and diagnostic delay were most certainly associated with worse outcome for the patient. Although the patient did not hold the local hospital responsible for the misdiagnosis, the case called attention to the need for greater vigilance in the presence of irregular pigmentation on the palmoplantar boundaries, where malignant melanoma should be considered. An important clue for avoiding misdiagnosis is that there is small irregular pigmentation on the edge of the nodule that can easily be mistaken for subcutaneous hemorrhage after incision, and biopsy is needed for differential diagnosis. Biopsy of the pigmentation showed tumor cells arranged in nests containing large amounts of melanin granules. It is also worth noting that amelanotic acral melanoma can be clinically and dermoscopically featureless. ${ }^{4}$ Awareness that amelanotic variants of acral melanoma can mimic the morphology of benign hyperkeratotic dermatoses may increase the rate of correct diagnosis and improve patient outcome. $\square$

\section{REFERENCES}

1. Thomas NE, Kricker A, Waxweiler WT, Dillon PM, Busman KJ, From L, et al. Comparison of clinicopathologic features and survival of histopathologically amelanotic and pigmented melanomas: a population-based study. JAMA Dermatol. 2014;150:1306-14.

2. Moreau JF, Weissfeld JL, Ferris LK. Characteristics and survival of patients with invasive amelanotic melanoma in the USA. Melanoma Res. 2013;23:408-13.

3. Lv J, Dai B, Kong Y, Shen X, Kong J. Acral Melanoma in Chinese: A Clinicopathological and Prognostic Study of 142 cases. Sci Rep. 2016;6:31432.

4. Thomas L, Phan A, Pralong P, Poulalhon N, Debarbieux S, Dalle S. Special locations dermoscopy: facial, acral, and nail. Dermatol Clin. 2013;31:615-24,ix.

AUTHORS'CONTRIBUTIONS
$\begin{array}{ll}\text { Wei Deng } & \text { (iD) ORCID 0000-0003-2924-9021 } \\ \text { Critical review of the manuscript } & \text { (iD) ORCID 0000-0002-4095-0903 } \\ \text { Ruixing Yu } & \text { (iD) ORCID 0000-0002-6314-3610 } \\ \text { Critical review of the literature } & \text { (iD) ORCID 0000-0002-8733-0402 } \\ \text { Yong Cui } & \\ \text { Critical review of the literature } & \\ \text { Zhancai Zheng } & \\ \text { Approval of the final version of the manuscript } & \end{array}$

How to cite this article: Deng W, Yu R, Cui Y, Zheng Z. Amelanotic acral melanoma misdiagnosed as verruca plantaris. An Bras Dermatol. 2019;94(1):86-8. 